\title{
OBJETO DE APRENDIZAGEM PARA SUPORTE AO APRENDIZADO DA DETERMINAÇÃO DE PARÂMETROS DE TRANSFORMADORES MONOFÁSICOS
}

\author{
Victoria A. S. HERRERA - victoria.herrera@fmu.br * \\ Guilherme H. EHARA- guihideuehara@gmail.com \\ Rafael T. NAKAYA - rafaeltuyoshi@gmail.com \\ André L. S. de SOUZA- andre.souza@cafbrasil.com.br \\ Yuri S. C. MOREIRA - yuristephen23@ gmail.com \\ Centro Universitário Faculdades Metropolitanas Unidas \\ Av. Brigadeiro Luís Antônio, 1089 - Bela Vista \\ CEP 01317-001 - São Paulo - SP
}

\begin{abstract}
Resumo: $O$ circuito equivalente do transformador em regime permanente serve como principal ferramenta de suporte para a determinação de parâmetros do equipamento. Existem procedimentos para obter parâmetros de transformadores monofásicos, especificamente ensaios a vazio e de curto circuito, que representam as condições extremas de funcionamento do equipamento. Conhecer os parâmetros do transformador é necessário não apenas para o estudo do seu comportamento, mediante o circuito equivalente, mas também para a determinação de perdas. Este trabalho tem como objetivo elaborar um objeto de aprendizagem que consiste em uma interface gráfica e interativa, para dar suporte ao aprendizado dos circuitos equivalentes do transformador e a interpretação correta dos dados obtidos nos ensaios clássicos para a determinação paramétrica. O público alvo do uso deste OA são estudantes de ensino superior, especificamente do curso de engenharia elétrica. O protótipo inicial deste objeto de aprendizagem foi aplicado em sala de aula apresentado uma avaliação favorável dos estudantes.
\end{abstract}

Palavras-chave: Transformador monofásico. Objetos de Aprendizagem. Determinação paramétrica. Ensaios clássicos.

\section{INTRODUÇÃO}

O transformador em regime permanente é um assunto estudado por todo futuro engenheiro elétrico, o funcionamento deste equipamento é razoavelmente simples, porém requer o entendimento do circuito equivalente, ferramenta que representa o transformador na forma de circuito elétrico com resistências e indutâncias. O transformador monofásico, objeto de estudo deste trabalho, conta com sete tipos de circuitos equivalentes: o circuito equivalente completo, e o circuitos T, L e o de ramo em série, cada um deles referidos ao lado de alta e baixa tensão.

Há dificuldade do entendimento deste assunto, devido à complexidade e abstração necessária para interpretar os diferentes tipos de circuito equivalente do transformador, e quando cada um deve ser usado, especificamente para a identificação dos parâmetros. Os valores dos parâmetros do transformador não podem ser obtidos mediante a medição direta, portanto, são determinados por meio de dois ensaios clássicos: ensaio a vazio e de curto circuito 
e posterior análise do circuito equivalente. Os ensaios não possuem uma alta complexidade, mas a dificuldade se encontra no momento de interpretar corretamente as medições e análise do circuito equivalente para este procedimento.

O presente trabalho tem como objetivo criar um objeto de aprendizagem, que contribua no entendimento da determinação de parâmetros para transformadores monofásicos. De acordo com Braga (2014a), objeto de aprendizagem (OA) é qualquer recurso digital que possa ser reutilizado ou referenciado na aprendizagem apoiada pela tecnologia. Na literatura, existem alguns trabalhos com objetivos de dar suporte ao ensino de máquinas elétricas (transformadores e motores) com foco no ensino superior, porém, não existem trabalhos que abordem especificamente o circuito equivalente e determinação paramétrica.

Na seção 2 deste trabalho apresenta-se a revisão bibliográfica e definição do problema, na seção 3 descreve-se a metodologia usada para o desenvolvimento do OA na tentativa de garantir uma interface gráfica amigável para contribuir ao aprendizado dos estudantes de engenharia elétrica. Na seção 4 apresentam-se os resultados e discussões, o protótipo inicial do OA e as avaliações dele após ter sido aplicado com um grupo de estudantes do sétimo semestre do curso de engenharia. Finalmente apresentam-se as considerações finais.

\section{REVISÃO BIBLIOGRÁFICA}

Os OAs fornecem um suporte no processo de aprendizado, e são de grande valia principalmente quando o assunto a ser estudado é complexo. Atualmente existe uma ampla produção de OAs, não só para ensino básico e médio, mas também para ensino superior na área das engenharias. Como exemplo, podem ser citados os trabalhos sem fins de lucrativos de Academy (2012), que tem diversos OAs com assuntos relacionados a leis de eletromagnetismo, motores CC e transformadores, e outros componentes eletrônicos, e, o trabalho de Holdings (2016), que possui um laboratório específico sobre transformadores, porém ele se concentra ao ensino da relação de tensões.

Considerando propostas mais complexas para estudo das máquinas elétricas de corrente alternada, existem trabalhos considerando transformadores (OLIVEIRA; CRUZ; LAUDARES, 2014) e (ELETROSYS, 2019) e motores de indução (BASU, 2015) e (LISITA; MACHADO; NERYS; FIGUEREDO; FIGUEREDO, 2010).

Oliveira, et al. (2014), apresenta um software livre que é um editor de áudio com a capacidade de gerar ondas (senoidais, quadradas, dente de serra e quadrada sem abas). Este trabalho foi desenvolvido com o propósito de tornar as aulas mais atrativas e significativas aos alunos, a vantagem de utilizar o software livre, reduzir custos e permitir acesso a todo público. O trabalho de Eletrosys (2019) realiza o dimensionamento do transformador, fornecendo os dados de tensões primária e secundária desejáveis, número de espiras, correntes e potência.

Para o estudo de motores elétricos, Lisita, et al. (2010), utilizam transdutores de tensão e corrente de alta precisão, para aquisição de dados e interface com o computador para assim determinar os parâmetros do motor, o trabalho não propõe especificamente um OA mas ele poderia ser usado para suporte ao estudo de determinação paramétrica de motores de indução. Por outro lado, Basu (2015), apresenta uma interface gráfica o MatLab/Simulink para realizar a determinação de parâmetros após obtenção das medições dos ensaios clássicos (ensaio CC, ensaio a vazio e ensaio de rotor bloqueado).

Considerando os trabalhos descritos anteriormente, observa-se que:

- Existem trabalhos que contribuem para o estudo de máquinas elétricas no nível superior, especificamente para a determinação de parâmetros, mas, eles estão focados nos motores de indução (BASU, 2015; LISITA, et al., 2010). 
- Os trabalhos e OAs existentes para estudar os transformadores, consideram questões mais simples, como a interação dos campos magnéticos (ACADEMY, 2012), a relação de tensões (HOLDINGS, 2016), questões relacionadas com o projeto de um transformador (ELETROSYS, 2019) ou estudo das propriedades magnéticas dos transformadores (OLIVEIRA; et al., 2014).

Com estas duas considerações, vista a ausência de um OA que possa ser reutilizado em sala de aula para o ensino do circuito equivalente do transformador monofásico, foi elaborado um OA que possa mostrar de forma gráfica e numérica a diferença entre os circuitos equivalentes, e dessa forma facilitar a internalização desse conteúdo.

O OA requer valores experimentais obtidos em laboratório, para realizar o processo de determinação dos parâmetros do transformador, sendo eles: a resistência do núcleo $\left(\mathrm{R}_{\mathrm{C}}\right)$, a indutância de magnetização $\left(X_{M}\right)$, a resistência equivalente $\left(R_{e q}\right)$ e a indutância equivalente $\left(\mathrm{X}_{\mathrm{eq}}\right)$; e complementarmente poder calcular as perdas no entreferro $\left(\mathrm{P}_{\text {núcleo }}\right)$ e perdas no cobre dos enrolamentos $\left(\mathrm{P}_{\mathrm{Cu}}\right)$. O objetivo do $\mathrm{OA}$ é que tenha uma interface gráfica intuitiva, garantindo ao aluno, preencher adequadamente as informações necessárias para obter os parâmetros, o OA realiza o cálculo dos parâmetros para 5 circuitos equivalentes do transformador: circuito completo, circuito em T (referido aos lados de alta e baixa tensão) e circuito em L (referido aos lados de alta e baixa tensão).

\section{METODOLOGIA USADA PARA O DESENVOLVIMENTO DO OA}

Uma vez definido o objetivo de elaborar um OA, para estudar os circuitos equivalentes do transformador, foi necessário adotar uma metodologia que considerasse tanto as necessidades pedagógicas quanto as tecnológicas. Nesse sentido, Braga (2014b), apresenta uma metodologia para desenvolvimento de OAs com foco na correta comunicação entre o professor (quem idealiza o OA) e o desenvolvedor. Dita metodologia conhecida como metodologia INTERA, tem oito etapas, neste trabalho consideraram-se apenas cinco: Contextualização, Requisitos, Arquitetura, Desenvolvimento, Teste e Qualidade, sendo descritas nas seções a seguir.

\subsection{Contextualização}

O OA será aplicado para o estudo de circuito equivalente de transformadores monofásicos, após os alunos estudarem os circuitos equivalentes e os ensaios para determinação de parâmetros, isto é, o OA não irá substituir os procedimentos e cálculos envolvidos neste assunto, e sim suporte ao aprendizado, permitindo que o aluno interaja com ele e possa realizar observações no seu próprio tempo. Os circuitos considerados pelo o OA são o circuito equivalente completo, os circuitos $\mathrm{T}$ (referidos ao lado de alta e baixa tensão) e os circuitos $\mathrm{L}$ (referidos ao lado de alta e baixa tensão), sendo um total de cinco circuitos.

\subsection{Requisitos}

Os requisitos desejáveis do OA se descrevem a seguir:

-Requisitos didático-pedagógicos: seguir a Teoria interacionista de Piaget (interação sujeito-objeto), permitindo que o estudante consiga explorar o objeto conforme a sua necessidade de aprendizado.

-Requisitos funcionais: Ter valores default, para o aluno verificar os cálculos que ele realiza com os resultados apresentados no OA, possibilidade de preenchimento de dados novos, resultados numéricos e gráficos que permitam verificar as diferenças entre cada circuito.

-Requisitos não funcionais: Confiabilidade dos resultados de acordo com 0 equacionamento para cada ensaio. Imagens de boa qualidade.

-Requisitos de usabilidade: fácil instalação, interface intuitiva, imagens 


\subsection{Arquitetura: Esboço}

Nesta etapa será descrita a arquitetura do OA mediante a elaboração de um esboço inicial, que permita ter uma ideia geral do resultado esperado do OA. Na Figura 1 apresenta-se o esboço representando as características descritas nos requisitos funcionais, isto é, uma seção com os exemplos genéricos, possibilidade de preenchimento de dados novos, resultados numéricos e gráficos em uma única tela.

Figura 1 - Esboço do Objeto de Aprendizagem.

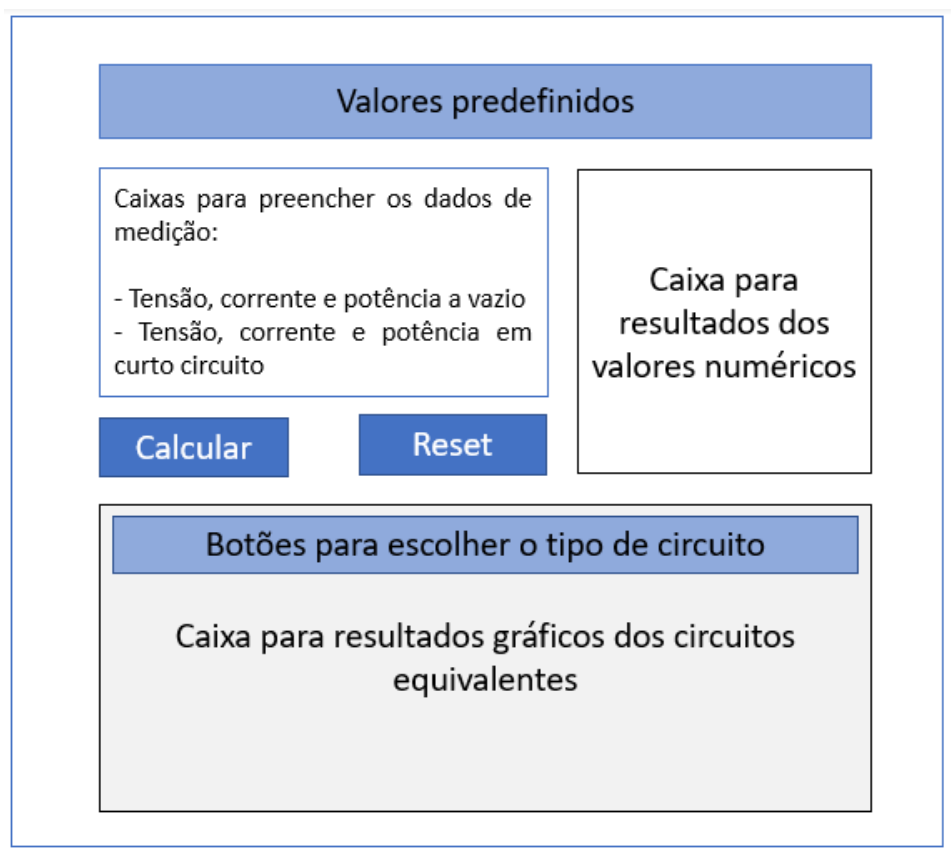

Fonte: elaboração própria

\subsection{Desenvolvimento}

A Figura 2 apresenta a síntese do desenvolvimento do trabalho mediante um fluxograma que descreve o correto funcionamento do AO. Nela observa-se que a sequência das ações, isto é deve iniciar através da inserção dos valores das medições dos ensaios a vazio, de curto circuito e a relação de transformação, estes valores devem ser inseridos de forma manual. A validação de preenchimentos certifica que todos os dados foram devidamente inseridos antes da etapa dos cálculos. Logo em seguida, os resultados são apresentados em uma lista de acordo com sua finalidade e posteriormente, são realocados para os circuitos equivalentes. Assim, os parâmetros são definidos e reorganizados de acordo com a necessidade de aplicação. Para facilitar a compreensão do funcionamento do OA foi desenvolvida a alternativa de utilizar valores genéricos já definidos.

Este OA foi desenvolvido por alunos de engenharia elétrica, autores deste artigo, como parte do TCC. O protótipo foi criado a partir do Microsoft Excel, que possui uma opção para desenvolvedores criarem programas utilizando o Microsoft Basic for Aplications. Nesta funcionalidade, o Excel permite a criação de módulos para o desenvolvimento da programação, onde todos os cálculos foram inseridos. Além dos módulos, também é possível criar formulários com botões, caixa de textos e outros elementos diversos que possibilitam a interação direta do usuário com a aplicação desejada. 
Figura 2 - Fluxograma do Objeto de Aprendizagem.

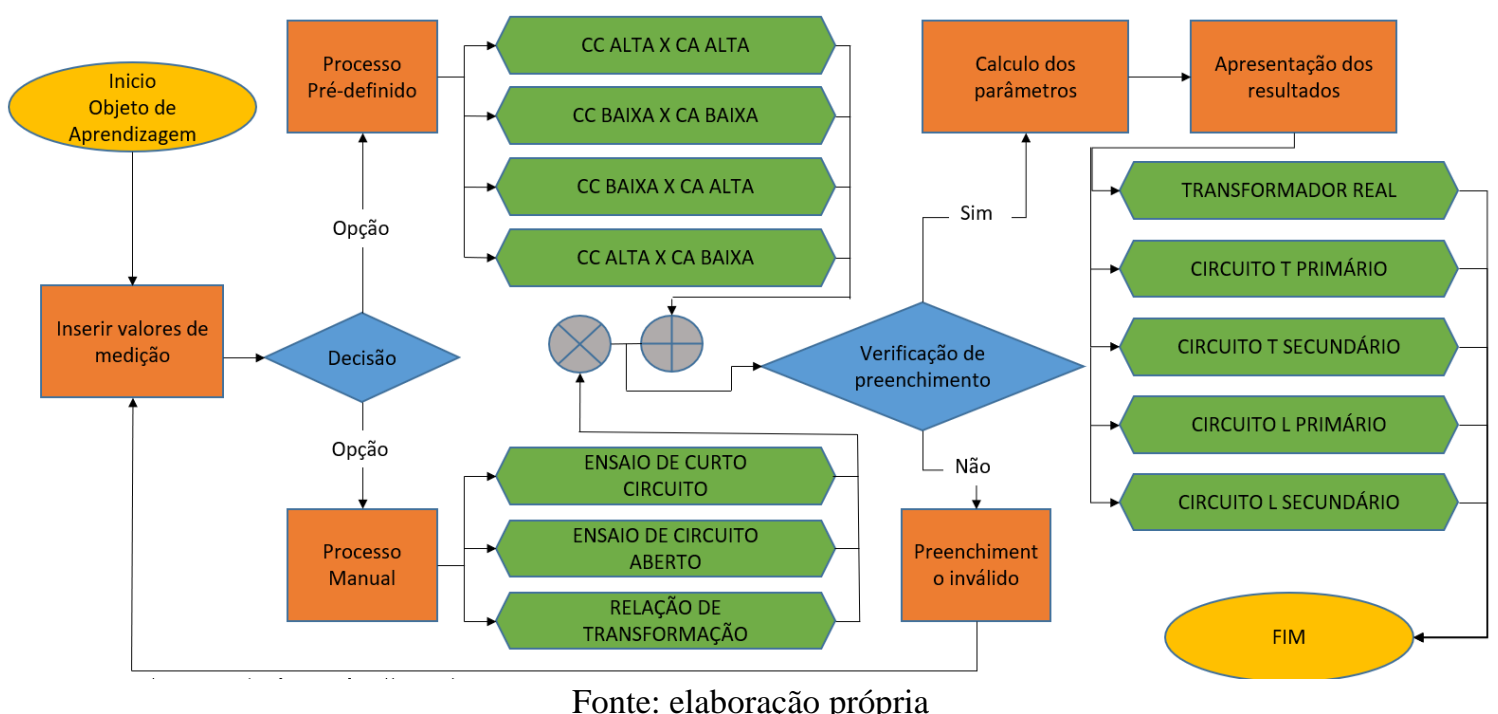

A fim de flexibilizar a usabilidade, foi inserida a opção para informar em qual lado, do primário ou do secundário, foram feitas as medições. Assim foram inseridos três botões adicionais nos valores pré-determinados para completar as combinações das medições. Também se viu necessário inserir um botão de "Reset" para limpar os valores inseridos anteriormente e preparar o formulário para uma nova utilização se necessário, e o botão de "Sair" para encerrar o uso após a utilização.

\subsection{Teste e Qualidade}

O OA foi apresentado aos estudantes descrevendo seus componentes, os locais onde podem ser preenchidos os dados, o armazenamento de alguns valores default obtidos da literatura, explicou-se o funcionamento geral e as janelas de resultados. Após, foi solicitado aos alunos resolverem um exercício usando o OA e finalmente preencheram um formulário anônimo com questões de usabilidade, questões sobre o aprendizado e sugestões.

\section{RESULTADOS E DISCUSSÃO}

Esta seção divide-se em duas subseções, a primeira dedicada a apresentar o protótipo do OA elaborado no Microsoft Excel, mediante o Microsoft Basic for Aplications, e a segunda descreve o procedimento de teste com usuários, isto é a aplicação em sala de aula.

\subsection{Resultados do protótipo inicial do OA}

A Figura 3 apresenta uma imagem do protótipo do OA. Na parte superior observam-se quatro botões que preenchem os campos de medição dos ensaios, com valores predefinidos de forma automática. Debaixo os valores default estão os campos de medição para os ensaios de curto circuito, circuito aberto (a vazio), a escolha do lado onde foi realizada a medição de cada ensaio e a relação de transformação. Na parte superior direita, encontram-se os campos com os resultados calculados a partir dos ensaios. Na parte inferior está localizado o circuito equivalente completo do transformador com os valores, de cada parâmetro, devidamente posicionados. Acima do circuito equivalente, é possível selecionar as opções para os outros tipos de representação do circuito (conforme a Figura 4). Na parte central localizam se os botões para as ações "Calcular", "Reset" e "Sair". 
Figura 3-Objeto de aprendizagem mostrando resultados.

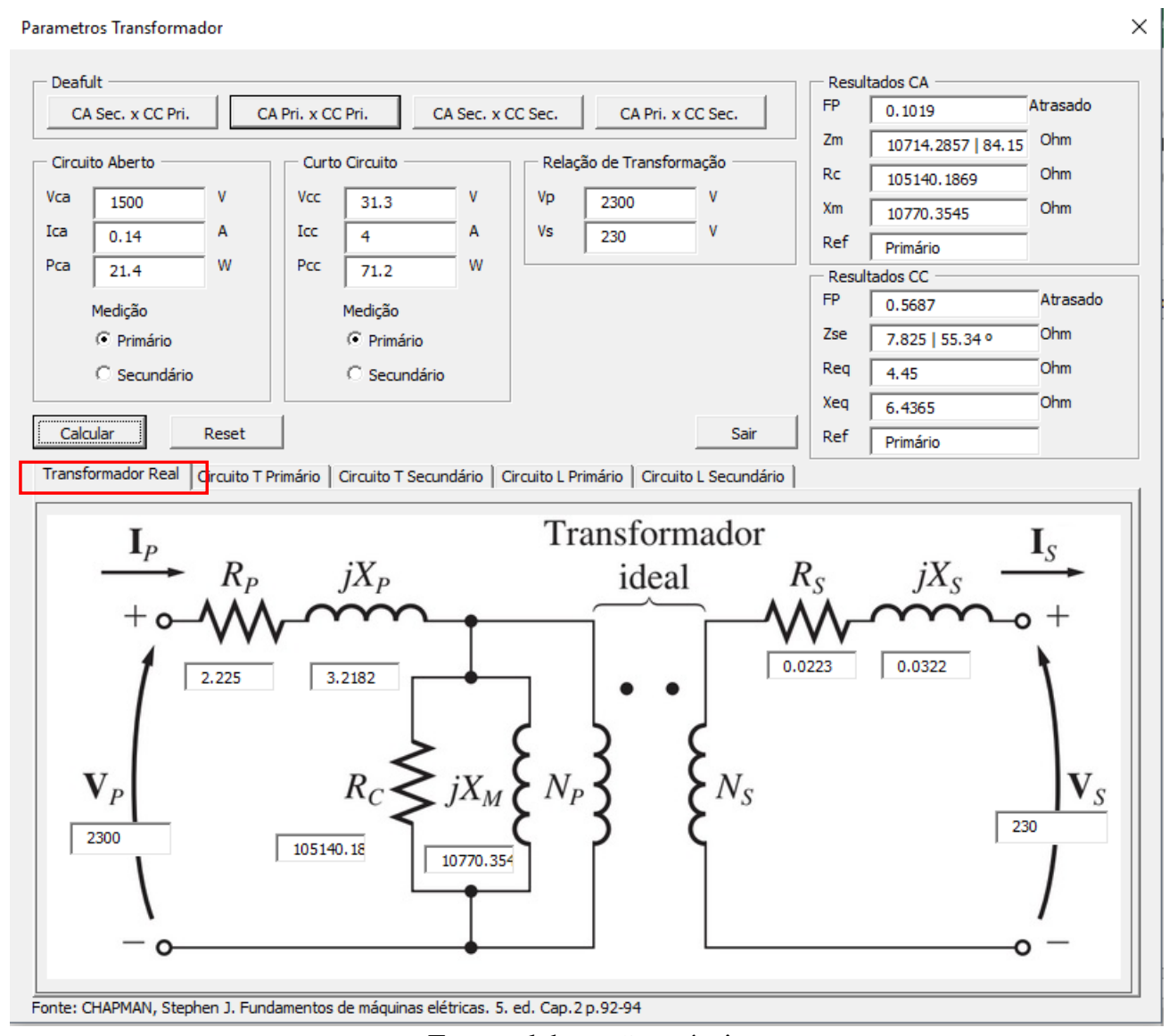

Fonte: elaboração própria

Na Figura 4 apresentam-se duas imagens menores do OA nas quais é possível observar, após clicar no botão de seleção, o circuito T referido ao lado primário (Figura 4a) e o circuito $\mathrm{L}$ referido ao lado secundário (Figura $4 \mathrm{~b}$ ).

\subsection{Aplicação do OA em sala de aula}

Em aulas prévias à interação com o OA, os alunos do sétimo semestre do curso de engenharia elétrica foram introduzidos ao assunto de circuitos equivalentes do transformador monofásico e os procedimentos sobre os ensaios clássicos. A explanação sobre os circuitos equivalentes foi realizada descrevendo o que cada parâmetro representa. Após, os alunos estudaram os procedimentos sobre os ensaios de curto circuito (para determinar $\mathrm{R}_{\mathrm{eq}}, \mathrm{X}_{\mathrm{eq}}$ e $\mathrm{P}_{\mathrm{Cu}}$ ) e circuito aberto (para determinar $\mathrm{R}_{\mathrm{C}}, \mathrm{X}_{\mathrm{M}}$ e $\mathrm{P}_{\text {núcleo }}$ ), sendo apresentado a eles o comportamento do circuito equivalente em cada ensaio, as conexões necessárias para realizar as medições mediante um infográfico. E foi realizado um exercício.

Com esses conhecimentos prévios dos alunos e, após um planejamento dos desenvolvedores do protótipo (alunos do décimo semestre do curso de engenharia elétrica autores deste artigo) e a professora (também autora), foi apresentado o OA em sala de aula. Após a interação individual com o OA, cada estudante preencheu um formulário com o objetivo de avaliar a usabilidade e alguns aspectos cognitivos gerais, o formulário foi elaborado em linguagem amigável e preenchido de forma anônima para fazer o processo mais descontraído garantindo respostas imparciais. 
CCOBENGE

"Os desafios para formar hoje o engenheiro do amanhã"

Figura 4 -Objeto de aprendizagem mostrando outros resultados.

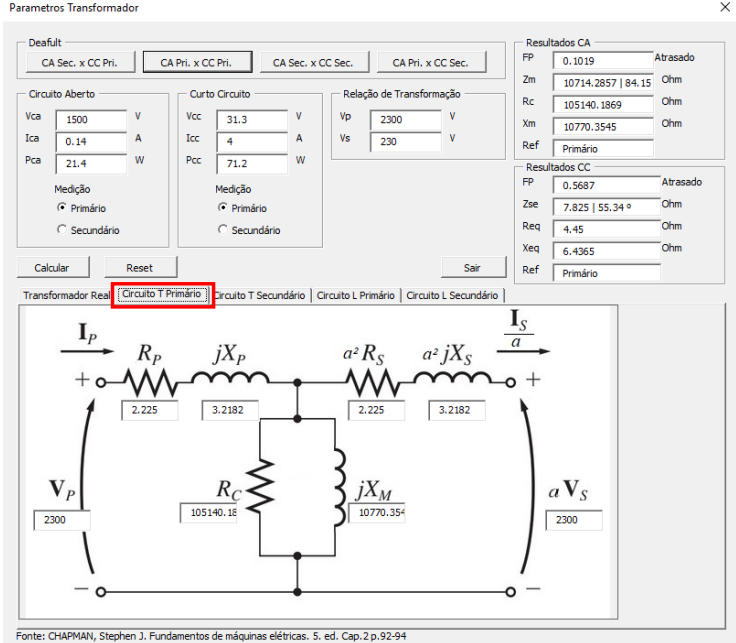

(a) Circuito $\mathrm{T}$ - lado primário

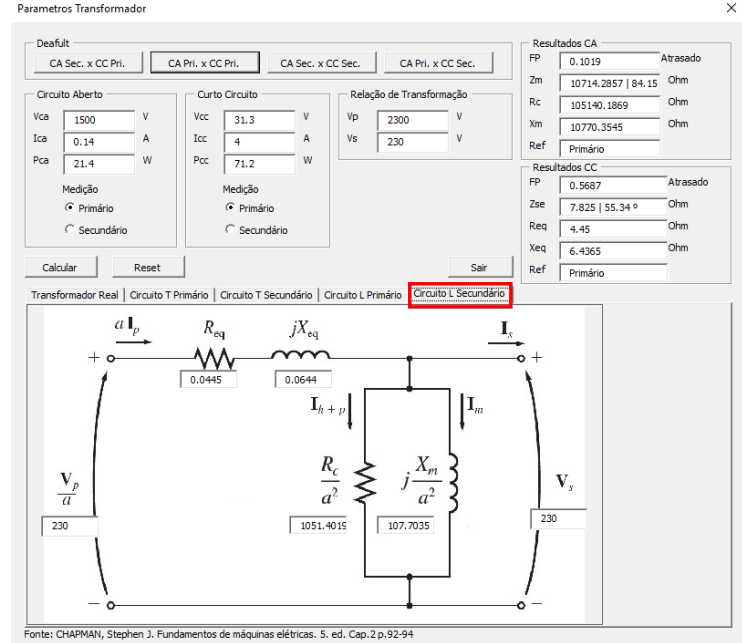

(b) Circuito L - lado secundário

Fonte: elaboração própria

Nas Tabela 1 a 3 apresenta-se o resumo das respostas dos estudantes. A Tabela 1 considera as questões relacionadas à usabilidade, sendo adotada a letra itálica para as perguntas e a letra normal para as opções apresentadas aos estudantes como resposta. A Tabela 2 considera as questões cognitivas, as questões realizadas aos estudantes estão também em itálico.

Observa-se na Tabela 1 e na Tabela 2 uma avaliação maioritariamente positiva, pretendese realizar esta experiência novamente no próximo semestre letivo, considera-se a possibilidade de inserir questões mais específicas para analisar melhor as dificuldades dos estudantes no aprendizado do circuito equivalente.

Finalmente apresenta-se a Tabela 3 com sugestões livres realizadas pelos usuários do AO, respondendo à questão "Se pudesse acrescentar alguma coisa no objeto de aprendizagem o que seria?".

Tabela 1 - Avaliação da usabilidade

\begin{tabular}{|l|c|}
\hline \multicolumn{1}{|c|}{ Como você avalia o uso inicial do objeto de aprendizagem? } & Avaliações \\
\hline Aceitável, consegui instalar com dificuldade e não deu erro. & $3,13 \%$ \\
\hline Bom, consegui instalar com facilidade. & $43,75 \%$ \\
\hline Ótimo, a instalação foi automática e rodou perfeitamente. & $53,12 \%$ \\
\hline $\begin{array}{c}\text { Como você avalia a apresentação e aparência do objeto de } \\
\text { aprendizagem? }\end{array}$ & Avaliações \\
\hline Aceitável, bonitinho (feio arrumado). & $6,25 \%$ \\
\hline Bom, está no caminho certo. & $78,13 \%$ \\
\hline Ótimo, é a oitava maravilha do mundo. & $15,62 \%$ \\
\hline Como você avalia a funcionalidade do objeto de aprendizagem? & Avaliações \\
\hline Aceitável, abri a tela de cálculos, mas não é muito intuitiva. & $12,25 \%$ \\
\hline Bom, abri a tela de cálculos e é bem intuitiva. & $50,00 \%$ \\
\hline Ótimo, muito intuitiva, facilitou a minha vida. & $37,50 \%$ \\
\hline
\end{tabular}


Tabela 2 - Avaliação cognitiva

\begin{tabular}{|l|c|c|c|}
\hline \multicolumn{1}{|c|}{ Perguntas } & $\begin{array}{c}\text { Mais ou } \\
\text { menos }\end{array}$ & Não & Sim \\
\hline $\begin{array}{l}\text { Antes do uso do objeto de aprendizagem você conseguia } \\
\text { visualizar a diferença entre os circuitos equivalentes? }\end{array}$ & $62,50 \%$ & $3,13 \%$ & $34,37 \%$ \\
\hline $\begin{array}{l}\text { Caso tenha respondido sim. Após interagir com objeto } \\
\text { de aprendizagem, você considera que ele ajudou a fixar } \\
\text { o conteúdo de forma gráfica? }\end{array}$ & - & $\begin{array}{c}16,62 \\
\%\end{array}$ & $84,38 \%$ \\
\hline $\begin{array}{l}\text { Caso tenha respondido não. Após interagir com o } \\
\text { objeto de aprendizagem você consegue visualizar a } \\
\text { diferença entre os circuitos equivalentes? }\end{array}$ & - & $\begin{array}{c}12,50 \\
\%\end{array}$ & $87,50 \%$ \\
\hline $\begin{array}{l}\text { Considera esta ferramenta útil para evitar confusões na } \\
\text { hora de construir o circuito equivalente completo? }\end{array}$ & - & $0,00 \%$ & $100,00 \%$ \\
\hline
\end{tabular}

Tabela 3-Observações dos alunos sobre o OA.

\begin{tabular}{|c|c|}
\hline Data/Hora & Se pudesse acrescentar alguma coisa no objeto de aprendizagem o que seria? \\
\hline $\begin{array}{c}11 / 05 / 2020 \\
19: 36: 10\end{array}$ & Projeto muito bem apresentado, fácil de absorver o conteúdo e praticar. \\
\hline $\begin{array}{c}11 / 05 / 2020 \\
19: 43: 31\end{array}$ & $\begin{array}{l}\text { o OA está bem completo, não vejo necessidade de mais nenhum } \\
\text { incremento. Parabéns. }\end{array}$ \\
\hline $\begin{array}{c}11 / 05 / 2020 \\
19: 46: 36\end{array}$ & $\begin{array}{c}\text { Achei o trabalho muito bacana! Facilita muito os cálculos e principalmente } \\
\text { a associação aos circuitos equivalentes. Seria bacana se tivesse uma tela } \\
\text { com os processos de cálculos, para estudantes seria importante para a } \\
\text { comparação de resultados, partes do processo com possíveis equívocos. } \\
\text { Parabéns pelo trabalho, ficou show de bola!!! ;D }\end{array}$ \\
\hline $\begin{array}{l}11 / 05 / 2020 \\
19: 51: 46\end{array}$ & $\begin{array}{c}\text { instalação do objeto muito simples e rápida, a apresentação dos alunos } \\
\text { [desenvolvedores do OA] na aula dever ser melhor apresentada, mais estão } \\
\text { no caminho correto, parabéns pelo projeto }\end{array}$ \\
\hline $\begin{array}{c}11 / 05 / 2020 \\
19: 58: 20\end{array}$ & $\begin{array}{l}\text { acredito que uma boa forma de melhorar a ferramenta seria introduzindo } \\
\text { uma ferramenta de legenda/cálculos quando passa o mouse em cima da } \\
\text { operação, se for um aplicativo para disponibilizar para pessoas com mais } \\
\text { dificuldades seria de grande ajuda, no pelo uso acredito que é uma } \\
\text { ferramenta muito útil para agilizar o cálculo no primário/secundário do } \\
\text { transformador. }\end{array}$ \\
\hline $\begin{array}{l}11 / 05 / 2020 \\
19: 58: 34\end{array}$ & Inserir opção de extração de relatório dos dados. \\
\hline $\begin{array}{l}11 / 05 / 2020 \\
20: 00: 34\end{array}$ & Que tal utilizar Power BI para parte gráfica, uma ótima ferramenta... \\
\hline $\begin{array}{l}11 / 05 / 2020 \\
20: 07: 46\end{array}$ & $\begin{array}{l}\text { Está muito intuitivo e claro para colocar e apresentar as informações, } \\
\text { facilita muito para visualizar e conferir os cálculos de forma rápida fácil }\end{array}$ \\
\hline $\begin{array}{l}11 / 05 / 2020 \\
20: 10: 32\end{array}$ & $\begin{array}{l}\text { Poderia ter um tópico de ajuda, quem está usando pela primeira vez fica um } \\
\text { pouco perdido. }\end{array}$ \\
\hline
\end{tabular}




\section{CONCLUSÕES E CONSIDERAÇÕES FINAIS}

O OA para o suporte ao estudo de circuitos equivalentes e determinação de parâmetros de transformadores monofásicos, foi idealizado pela professora das disciplinas Conversão eletromecânica de energia e Máquinas elétricas, devido a verificar, no decorrer dos semestres, a dificuldade dos alunos para conseguir absorver estes conceitos. Vista esta necessidade, o OA foi desenvolvido pelos alunos do décimo semestre como projeto de TCC no Microsoft Excel, mediante o Microsoft Basic for Aplications.

A etapa de teste do OA mostrou uma avaliação positiva por parte dos usuários, durante a aula observou-se que os estudantes prestaram atenção à explicação realizada pelos desenvolvedores e executaram com prontidão os testes no OA. Esta ferramenta pode também suprir uma eventual ausência de laboratório. As sugestões de melhorias que os estudantes realizaram tem como foco o aprimoramento na usabilidade, porém pôde-se verificar que o OA não se desviou dos objetivos pedagógicos.

Após a aplicação do OA em sala de aula, foram verificadas duas ações futuras, uma delas considerando o aspecto de aprendizado dos estudantes e a outra o aspecto técnico. Quanto ao aspecto de aprendizado, se faz necessária uma avaliação posterior dos estudantes, verificar por exemplo a porcentagem que consegue responder corretamente este tipo de exercício nas provas, comparado com turmas que realizam a prova sem ter usado o OA. Quanto ao aspecto técnico, os autores têm como objetivo dar continuidade ao trabalho na procura do uso de um software livre ou até a possibilidade de criar um aplicativo para celular que realize o mesmo processo, fazendo o OA ainda mais atrativo aos estudantes. As sugestões levantadas pelos usuários nesta avaliação inicial são consideradas de grande valia na continuidade deste trabalho.

\section{Agradecimentos}

Ao Centro Universitário Faculdades Metropolitanas Unidas, ao Prof. Dr. René Teixeira (coordenador do curso) pelas sugestões durante a disciplina TCC, ao colega Elias da Silva pela colaboração.

\section{REFERÊNCIAS}

ACADEMY, Magnet. Transformers: interactive tutorials. Interactive tutorials. 2012. Disponível em: https://nationalmaglab.org/education/magnet-academy/watchplay/interactive/transformers. Acesso em: 04 maio 2020.

BASU, Jayanta Bhusan. MATLAB based performance prediction of a three-phase induction motor utilising its test results. International Journal Of Engineering Research \& Technology (ijert). India, p. 8-10. 09 set. 2015.

BRAGA, Juliana org. Objetos de Aprendizagem: Introdução e fundamentos. Vol. $1,1^{\mathrm{a}}$ ed. Santo André: Editora da UFABC, 2014a.

BRAGA, Juliana org. Objetos de Aprendizagem: Metodologia de desenvolvimento. Vol. 2,1 ${ }^{\mathrm{a}}$ ed. Santo André: Editora da UFABC, 2014b.

CHAPMAN, Stephen J. Fundamentos de máquinas elétricas. $5^{\mathrm{a}}$ ed. Porto Alegre: The Mcgraw-hill Companies, 2013. 684 p. Tradução de Anatólio Laschuk. 
ELETROSYS, Eletrônica. PROGRAMA PARA CALCULO DE TRANSFORMADORES. 2019. Disponível em: https://eletrosys.blogspot.com/2018/04/transformadores-programa-paracalculo.html. Acesso em: 14 nov. 2019.

HOLDINGS, Mc Graw Hill Global Education (ed.). Virtual Lab: how a transformer works?. How a transformer works? 2016. Disponível em: http://www.glencoe.com/sites/common_assets/science/virtual_labs/E15/E15.html. Acesso em: 04 maio 2020.

OLIVEIRA, Bruno Randal de et al. Estudando Propriedades de Transformadores Elétricos com Software Livre. 2014. 66 f. Tese (Doutorado) - Curso de Engenharia Elétrica, Uefs, Rio de Janeiro, 2014. Cap. 01.

UMANS, Stephen D. Máquinas elétricas de Fitzgerald e Kingsley. 7. ed. Porto Alegre: The Mcgraw-hill Global Education Holdings, 2014. 708 p. Tradução de Anatólio Laschuk.

SEIDL, Martina; BRANDSTEIDL, Marion; KAPPEL, Gerti; HUEMER, Christian. UML @ Classroom: an introducction to object - oriented modeling. Switzerland: Springer, 2015.

LINTZMAYER, Carla Negri; MOTA, Guilherme Oliveira. Análise de Algoritmos e Estruturas de Dados. 2020. 295 f. Tese (Doutorado) - Curso de Engenharia Elétrica, Análise de Algoritmo, Universidade Federal do Abc, Santo André, 2020.

\section{LEARNING OBJECT FOR SUPPORTING THE STUDY OF PARAMETER DETERMINATION PROCESS OF SINGLE-PHASE TRANSFORMERS}

Abstract: The steady state transformer equivalent circuit is a useful tool for parameter determination. It is necessary to know the parameters of the transformer not only for studying its behavior, through the equivalent circuit, but also for determining losses. There are procedures parameter determination of a single-phase transformer, specifically short and open circuit tests, which represents the extreme operating conditions of the equipment. The parameters of the transformer are necessary not only for studying its behavior, by means of the equivalent circuit, but also for determining losses. This work aims to elaborate a learning object which consists on a graphic and interactive interface, to support the learning of the equivalent circuits of the transformer and the correct interpretation of the data obtained from classical tests. The learning object target audience is higher education students, specifically from electrical engineering courses. The initial prototype of the learning object was applied at the classroom, showing a favorable evaluation of the students.

Keywords: Single-phase transformer; Learning Objects. Parametric determination. Classic experimental test. 